\title{
Development and application of a TaqMan-MGB real-time RT-PCR assay for the detection of porcine epidemic diarrhoea virus strains in China
}

\author{
Yi-Xuan Hou ${ }^{1}$, Chun Xie ${ }^{1}$, Kang Wang ${ }^{2}$, Yu-Ting Zhao ${ }^{1}$, \\ Yang-Yang Xie ${ }^{1}$, Hong-Yan Shi ${ }^{3}$, Jian-Fei Chen ${ }^{3}$, Li Feng ${ }^{3}$, \\ Guang-Zhi Tong ${ }^{2}$, Xiu-Guo Hua ${ }^{1}$, Cong-Li Yuan ${ }^{1}$, \\ Yan-Jun Zhou ${ }^{2}$, Zhi-Biao Yang ${ }^{1}$ \\ ${ }^{1}$ Shanghai Key Laboratory of Veterinary Biotechnology, School of Agriculture and Biology, \\ Shanghai Jiao Tong University, Shanghai 200240, People's Republic of China \\ ${ }^{2}$ Division of Swine Infectious Diseases, \\ Shanghai Veterinary Research Institute of Chinese Academy of Agricultural Sciences, \\ Shanghai 200241, People's Republic of China \\ ${ }^{3}$ Division of Swine Infectious Diseases, National Key Laboratory of Veterinary Biotechnology, \\ Harbin Veterinary Research Institute of Chinese Academy of Agricultural Sciences, \\ Harbin 150001, People's Republic of China \\ yjzhou@shvri.ac.cn
} Received: September 9, 2015 Accepted: April 22, 2016

\begin{abstract}
Introduction: A real-time RT-PCR method for identification and quantification of porcine epidemic diarrhoea virus (PEDV) strains in China was developed. Material and Methods: Based on the conserved sequence of the PEDV nucleocapsid (N) gene, a primer pair and probe were designed to establish a TaqMan-MGB real-time RT-PCR assay for quantitative detection of the virus. The sequence was cloned into the pMD18-T vector and a series of diluted recombinant plasmids were used to generate a standard curve with an R2 value of 0.999. Results: The developed quantitative PCR assay detected viral titres as low as $0.1 \mathrm{TCID}_{50}$ with high specificity and no cross-reaction with other porcine viruses (PoRV, TGEV, PRRSV, or CSFV). The intra-batch and inter-batch coefficients of variation were both less than $1 \%$, which indicated good reproducibility. Thirty clinical diarrhoea samples obtained from pigs in Shanghai and Fujian were analysed using this quantitative PCR assay. Out of these samples, 93.3\% were found to be PEDV positive. Conclusion: This approach is suitable for clinical sample identification and pathogenesis studies.
\end{abstract}

Keywords: porcine epidemic diarrhoea virus, TaqMan-MGB real-time RT-PCR, People's Republic of China.

\section{Introduction}

The first case of porcine epidemic diarrhoea (PED) was reported in the UK in 1971 (14). The disease was caused by the porcine epidemic diarrhoea virus (PEDV). Outbreaks of PED are seasonal, usually occur in autumn and spring, and result in a high mortality among infected piglets (4). Clinical symptoms of PED are: watery diarrhoea, vomiting, and weight loss (22). PED is spreading by faecal-oral transmission and is contagious for newborn piglets within the first week, with a high mortality rate of approximately $90 \%-100 \%$ due to extremely watery diarrhoea (13).

PEDV is an alphacoronavirus, comprising an enveloped positive-strand RNA (2). The genome of PEDV is approximately $28 \mathrm{~kb}$, with seven open reading frames (ORFs) encoding release complex, spike, membrane, small membrane, and envelope proteins (17). Since 2010, outbreaks of a new and highly virulent PED have spread across China, showing various mutations of $\mathrm{N}$-terminus of $\mathrm{S}$ gene. As 
polygenetic analysis demonstrated, there are at least two prevalent genogroups of PEDV across China (3, 5, $6,11-13,23,27)$. Because of the low adaptation to cell culture, it is difficult to differentiate PEDV from other enteric viral pathogens using cell culture isolation (16). In addition, due to the high frequency of mutations, various PEDV epidemic strains cannot be correctly identified using conventional primers for PCR assay (1). Real-time RT-PCR with specific primer pair and probe is a more specific and sensitive technique for the identification of the presence of the virus in field samples, and also provides the capacity to quantify the virus (15). Similar assays have been developed for PEDV detection of prevalent Korean PEDV by designing primers against $\mathrm{N}$ gene (10) and against $\mathrm{S}$ gene of Chinese-prevalent strains (26). In the presented study, we established a real-time RT-PCR method that can be used to identify and quantify both classical and epidemic PEDV strains in China. In order to detect both prevalent strains, we developed a rapid and sensitive TaqMan real-time RT-PCR assay based on highly conserved sequence on $\mathrm{N}$ gene. The results of this assay indicate that they are accurate and rapid, and thus they have proven to be a useful tool for studying epidemiology and phenotypic properties of PEDV.

\section{Material and Methods}

Clinical samples, viruses, and cell lines. Porcine faecal samples were obtained from pig farms in Fujian Province and Shanghai Municipality, China. PEDV HLJ/2011 strain, porcine reproductive and respiratory syndrome virus (PRRSV) HuN4-F112 strain, transmissible gastroenteritis virus (TGEV), classical swine fever virus (CSFV), porcine rotavirus (PoRV), and Vero cell lines were from the collection of the Zoonosis and Comparative Medicine Laboratory, Shanghai Jiao Tong University.

Reagents and equipment. The following reagents were used: ExTaq DNA polymerase, dNTPs, pMD18-T plasmid (TaKaRa, Japan); RNeasy kit (Qiagen, Germany), reverse transcription kit (Thermo, USA), plasmid extraction kit, and agarose gel DNA extraction kit (Omega, USA). E. coli DH5 $\alpha$ was from the collection of our laboratory. Tests were performed on a Veriti 96-well thermal cycler (Applied Biosystems, USA) and a Mastercycler ep realplex (Eppendorf, Germany).

Primers and probes. The nucleocapsid $(\mathrm{N})$ gene sequences of seven different PEDV strains (CV777, $\mathrm{Brl} / 87, \mathrm{CH} / \mathrm{S}, \mathrm{ZJCZ} 4, \mathrm{CHGH} 1, \mathrm{BJ}-2012-1$, and $\mathrm{HLJ} / 2012$ ) were aligned using Mega 5 software, and Oligo 6 software was used to design a pair of primers for amplification of a $169 \mathrm{bp}$ PCR product based on the conserved sequence of the $\mathrm{N}$ gene of PEDV ZJCZ4 (GenBank No. JX524137). A probe sequence was designed based on a sequence between the forward and reverse primers, with FAM fluorescence group at the
5 '-end and an MGB quenching group at the 3 -end. The sequences of the primers and probe were as follows: forward primer (PEDV-U): 5'-ATGGCAACAATA GGTCTA3'; reverse primer (PEDV-L): 5'-GAC TGGTTCCTGTTATTG-3'; probe: FAM-CCAAGT AACAACAGAGGCAATAACCAG-MGB.

Preparation of RNA transcript controls. Supernatants of cultured Vero cells inoculated with PEDV HLJ/2011 strain were centrifuged at $10000 \times \mathrm{g}$ for $10 \mathrm{~min}$. Viral RNA was prepared from $140 \mu \mathrm{L}$ of the supernatant using the RNeasy ${ }^{\circledR}$ Viral RNA kit (Qiagen, Germany) according to the manufacturer's instructions with the following slight modification: the viral RNA was eluted in $50 \mu \mathrm{L}$ of $\mathrm{H}_{2} \mathrm{O}$. Total RNA was prepared from faecal specimens using the RNeasy ${ }^{\circledR}$ Viral RNA kit (Qiagen, Germany); the samples were diluted $(\times 100)$ in phosphate buffered saline following the manufacturer's instructions.

Reverse transcription of the prepared total RNA was performed with RevertAid First Strand cDNA Synthesis kit (Thermo, USA) using PEDV-U and PEDV-L as primers to amplify the target sequence. The following RT-PCR conditions were used: initial denaturation for $5 \mathrm{~min}$ at $95^{\circ} \mathrm{C}$, with a subsequent 45-cycle amplification comprising denaturation at $95^{\circ} \mathrm{C}$ for $30 \mathrm{~s}$, annealing at $50^{\circ} \mathrm{C}$ for $30 \mathrm{~s}$, and extension at $72^{\circ} \mathrm{C}$ for $1 \mathrm{~min}$. The target DNA sequence was purified from the PCR products, ligated into the pMD18T plasmid (TaKaRa, Japan), and transformed into $E$. coli DH5a strain. After blue-white screen and confirmation by PCR, reference plasmid was extracted with a plasmid extraction kit (Omega, USA) and the plasmid copy number was titrated by NanoDrop 2000 UV-Vis Spectrophotometer (Thermo, USA).

Reference plasmid was 10 times diluted with $\mathrm{dH}_{2} \mathrm{O}$ to prepare solutions at concentrations from $10^{-3}$ to $10^{-7}$ copies $/ \mu \mathrm{L}$. The $\mathrm{TCID}_{50}$ value of the virus was titrated and calculated by Reed and Muench method (18). Dilutions $\left(10^{6}\right.$ to $\left.10^{-1} \mathrm{TCID}_{50}\right)$ of the virus were prepared in $\mathrm{H}_{2} \mathrm{O}$ before total RNA was isolated and reverse transcribed as described earlier in this section.

Real-time PCR condition. One microlitre of reference plasmid (from $1.53 \times 10^{9}$ to $1.53 \times 10^{3}$ copies/ $\mu \mathrm{L}$ dilutions) was used as a template in the realtime PCR assay, mixed with $2.5 \mu \mathrm{L}$ of $10 \times$ ExTaq Buffer $\left(\mathrm{Mg}^{2+}\right.$ plus), $2.5 \mu \mathrm{L}$ of dNTP mixture $(2.5 \mathrm{mM}$ each), $1 \mu \mathrm{L}$ of PEDV-U and PEDV-L $(250 \mu \mathrm{M}), 0.2 \mu \mathrm{L}$ of Ex Taq $(5 \mathrm{U} / \mu \mathrm{L}), 0.5 \mu \mathrm{L}$ of probe $(10 \mathrm{pmol} / \mu \mathrm{L})$, and 16.3 $\mu \mathrm{L}$ of $\mathrm{ddH}_{2} \mathrm{O}$. The following amplification conditions were used: initial denaturation for $2 \mathrm{~min}$ at $94^{\circ} \mathrm{C}$, with subsequent amplification (40 cycles) comprising denaturation at $94^{\circ} \mathrm{C}$ for $20 \mathrm{~s}$, and annealing at $50^{\circ} \mathrm{C}$ for $1 \mathrm{~min}$.

Conventional PCR condition. To compare the accuracy of diagnosis, a conventional PCR using primers specific for $\mathrm{N}$ gene was used for testing 30 field faecal samples. Total RNA extraction and reverse transcription were described above. One microlitre of total RNA was used for PCR amplification by Taq 
polymerase (TaKaRa, Japan) with primers: forward primer (NU): 5'-GGTACTTGC AAACAATGCTGT-3'; reverse primer (NL): 5'-TCT GGGATGTCTTTA AGGTCA-3', enabling to obtain the product of a 740 bp fragment. After PCR amplification, samples were loaded in $1 \%$ agarose gel for electrophoresis.

Statistical analysis. Statistical analysis referred to the minimum number of copies per microlitre of constructed reference plasmid that can be measured in this assay, comparing $\mathrm{Ct}$ value to negative control. Specificity referred to detecting PEDV from several different swine viral total cDNA samples, by comparing their $\mathrm{Ct}$ value as well. Repeatability referred to the precision and robustness of the assay using different dilutions of the same reference plasmid repeatedly. One microlitre of each reference plasmid sample in $10^{5}, 10^{6}$, and $10^{7}$ copies $/ \mu \mathrm{L}$ was used as template for this assay; each sample was measured three times and mean and standard deviation of $\mathrm{Ct}$ values were calculated.

\section{Results}

Development of real-time RT-PCR standard curves. A standard curve was generated by the Veriti 96-well thermal cycler after 40 cycles of reference plasmid reaction. Each point represents $\mathrm{Ct}$ value of different concentration of reference plasmid, from 1.53 $\times 10^{9}$ to $1.53 \times 10^{3}$ copies/ $\mu \mathrm{L}$ dilutions (Figs 1,2 ). Equation of this standard curve is $\mathrm{Y}=-3.358 \operatorname{LogX}+$ $37.49\left(R^{2}=0.999\right.$, efficiency $\left.=0.98\right)$.

Sensitivity evaluation. The analysis of total RNA of PEDV-HLJ/2011 in the range from $10^{6}$ to $10^{-1}$ TCID $_{50}$ showed that this assay has the capacity to detect virus sample with a minimum $10^{-1} \mathrm{TCID}_{50}$ titre (Fig. 3).

Specificity evaluation. Using PoRV, TGEV, PRRSV, PEDV-HLJ/2011 and non-infected Vero cells total RNA as templates in real-time RT-PCR assays it was shown that only PEDV cDNA was detected, while amplification of cDNA from other viruses and noninfected Vero cells cDNA was negative (Fig. 4).

Repeatability evaluation. Real-time RT-PCR assay was used to test $10^{5}, 10^{6}$, and $10^{7}$ copies of the plasmid samples in three independent experiments. The low intra- and inter-batch standard deviation (SD) and coefficient of variation $(\mathrm{CV})$, which was less than $5 \%$ of the results obtained, indicated that this assay has good repeatability (Table 1 ).

Diagnostic accuracy. Thirty faecal samples were tested using the PEDV-U/PEDV-L primer pair for conventional or real-time RT-PCR assays. Using the conventional RT-PCR method, 25 (83.3\%) samples were identified as PEDV positive, while $28(93.3 \%)$ samples were identified as PEDV positive using the real-time RT-PCR method (Figs 5, 6).

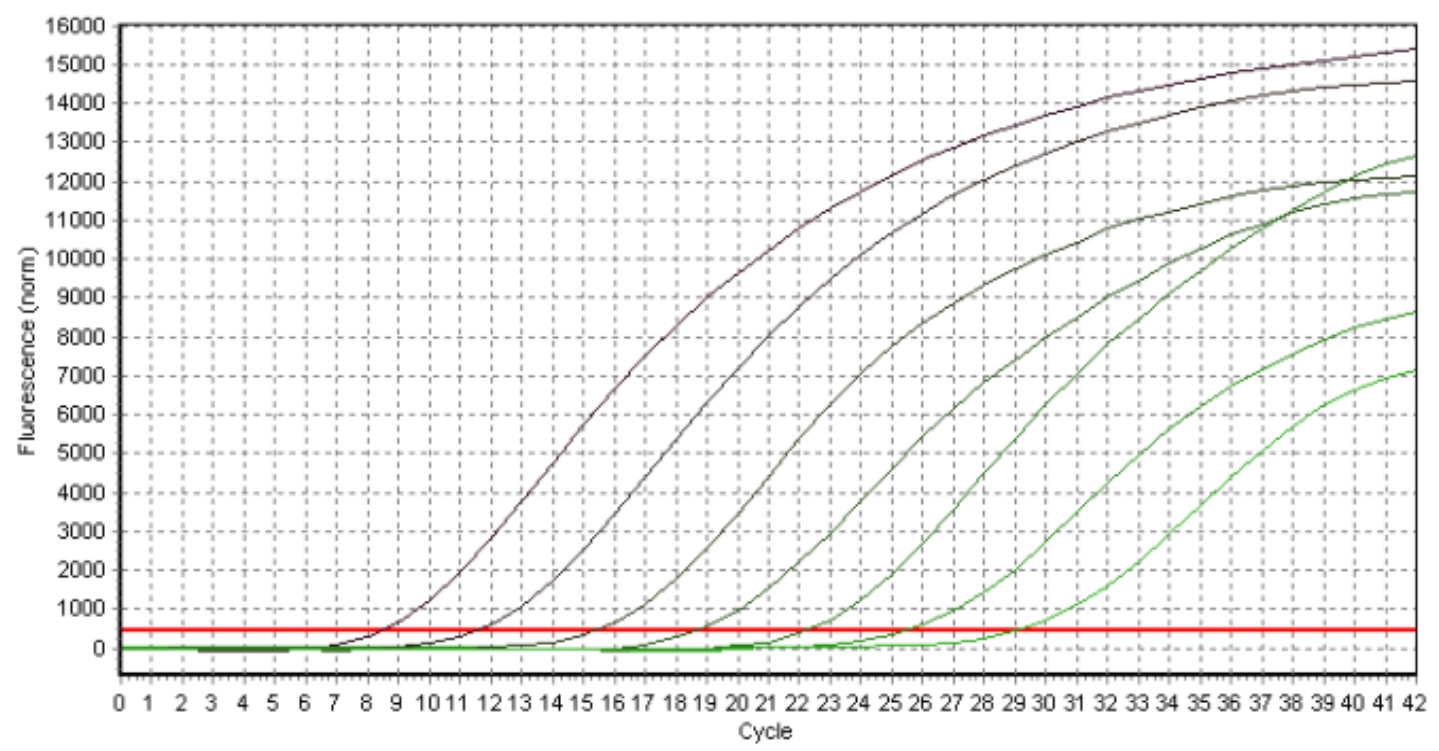

Fig. 1. TaqMan-MGB fluorescent quantitative RT-PCR amplification plots for PEDV detection (left to right: $1.53 \times 10^{9}-1.53 \times 10^{3}$ copies of reference plasmid)

Table 1. Repeatability evaluation of TaqMan-MGB real-time RT-PCR assay

\begin{tabular}{lllllllll}
\hline \multirow{2}{*}{ Samples } & \multirow{2}{*}{ Copies } & \multirow{2}{*}{$\begin{array}{l}\text { Repeat } \\
\text { times }\end{array}$} & \multicolumn{2}{l}{ Intra-batch Ct value } & \multicolumn{3}{c}{ Inter-batch Ct value } \\
\cline { 3 - 8 } & & Mean & SD & CV (\%) & Mean & SD & CV (\%) \\
\hline Sample 1 & $10^{7}$ & 3 & 18.28 & 0.85 & 4.6 & 18.76 & 0.32 \\
Sample 2 & $10^{6}$ & 3 & 21.35 & 0.71 & 3.3 & 21.47 & 0.66 \\
Sample 3 & $10^{5}$ & 3 & 25.21 & 0.93 & 3.7 & 25.24 & 0.72 & 2.9 \\
\hline
\end{tabular}




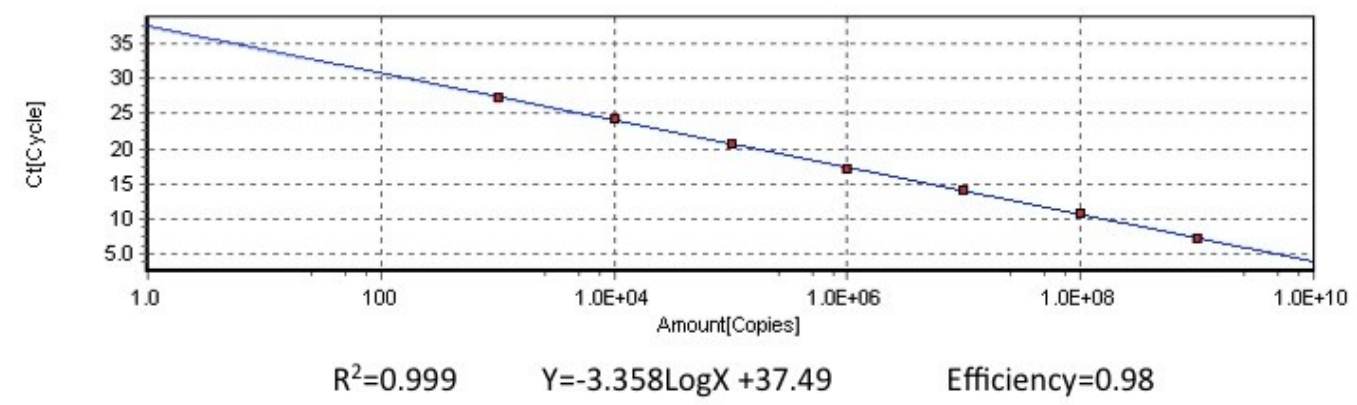

Fig. 2. Standard curve of TaqMan-MGB fluorescent quantitative RT-PCR for PEDV detection (left to right: $1.53 \times 10^{9}-$ $1.53 \times 10^{3}$ copies of reference plasmid)

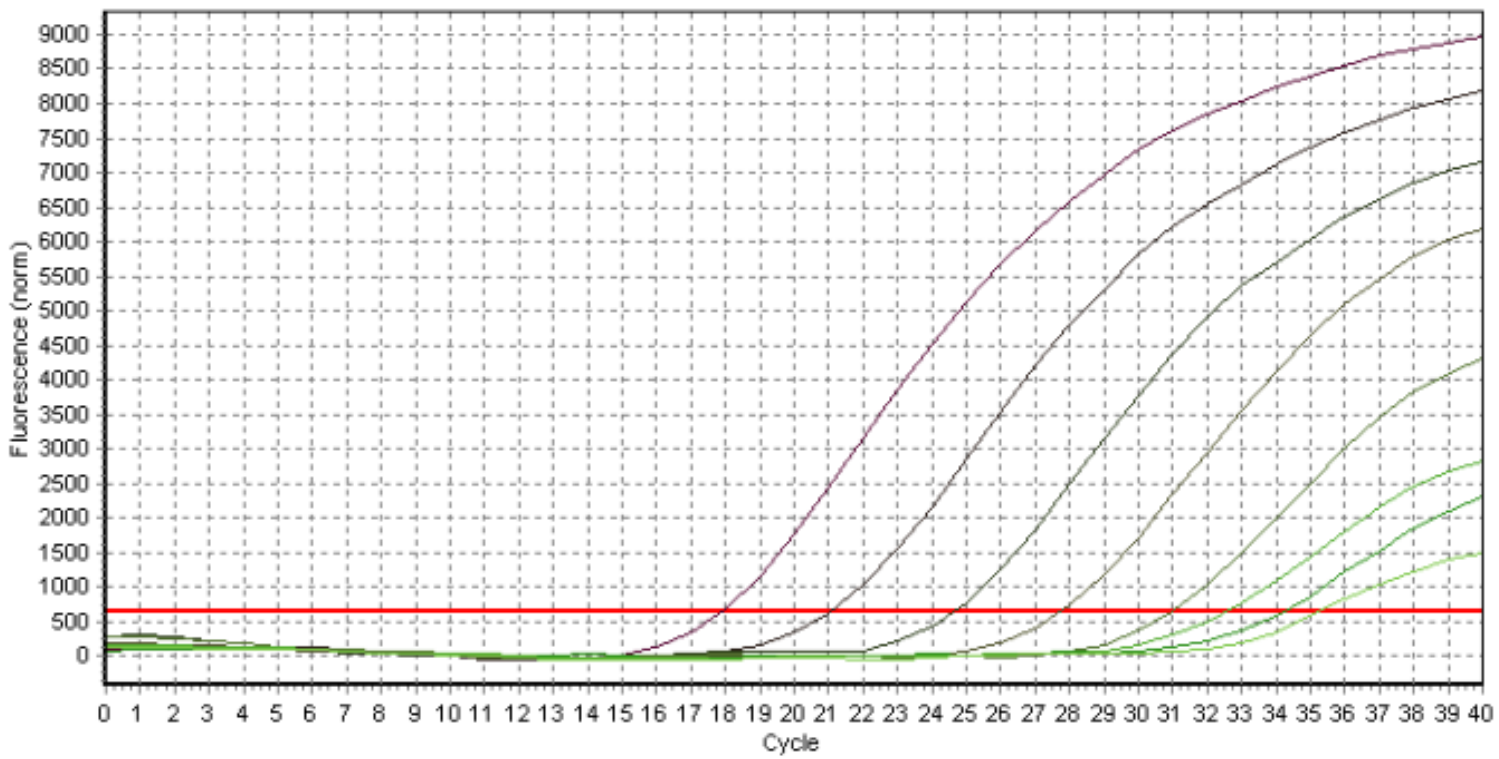

Fig. 3. Sensitivity evaluation of TaqMan-MGB fluorescent quantitative RT-PCR for PEDV detection (left to right: $10^{6}-10^{-1} \mathrm{TCID}_{50}$ titre of PEDV cDNA)

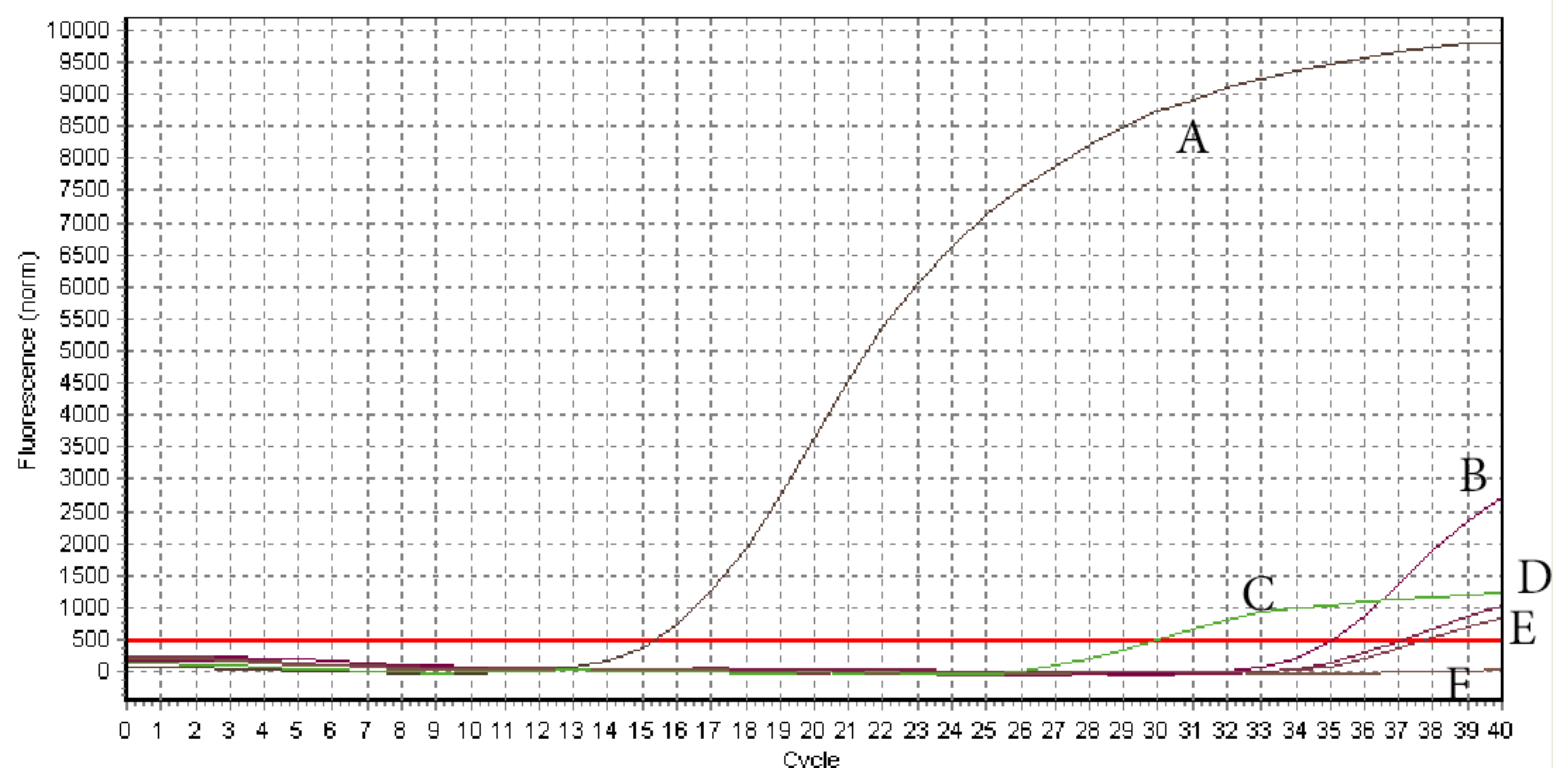

Fig. 4. Specificity evaluation of TaqMan-MGB fluorescent quantitative RT-PCR for PEDV detection (A: PEDV; B: PoRV; C: TGEV; D: CSFV; E: PRRSV; F: Vero cells) 


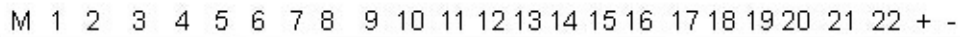

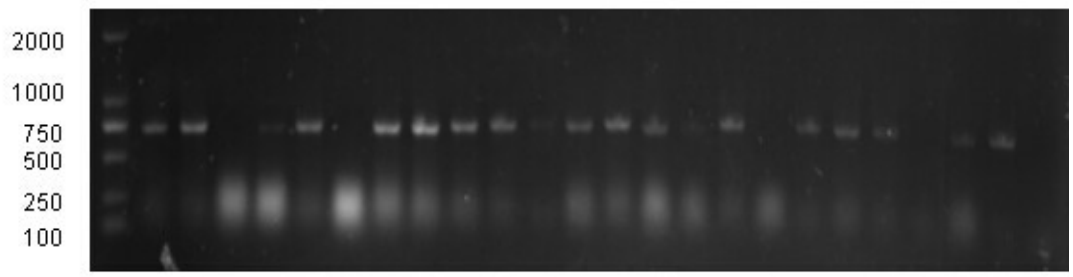

M $2324252627282930+-$

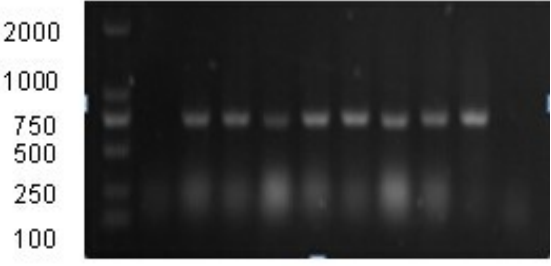

Fig. 5. Conventional PCR analysis of clinical samples (M: DNA Marker DL2000; Lanes 1-30: faecal samples; +: positive control; -: negative control)

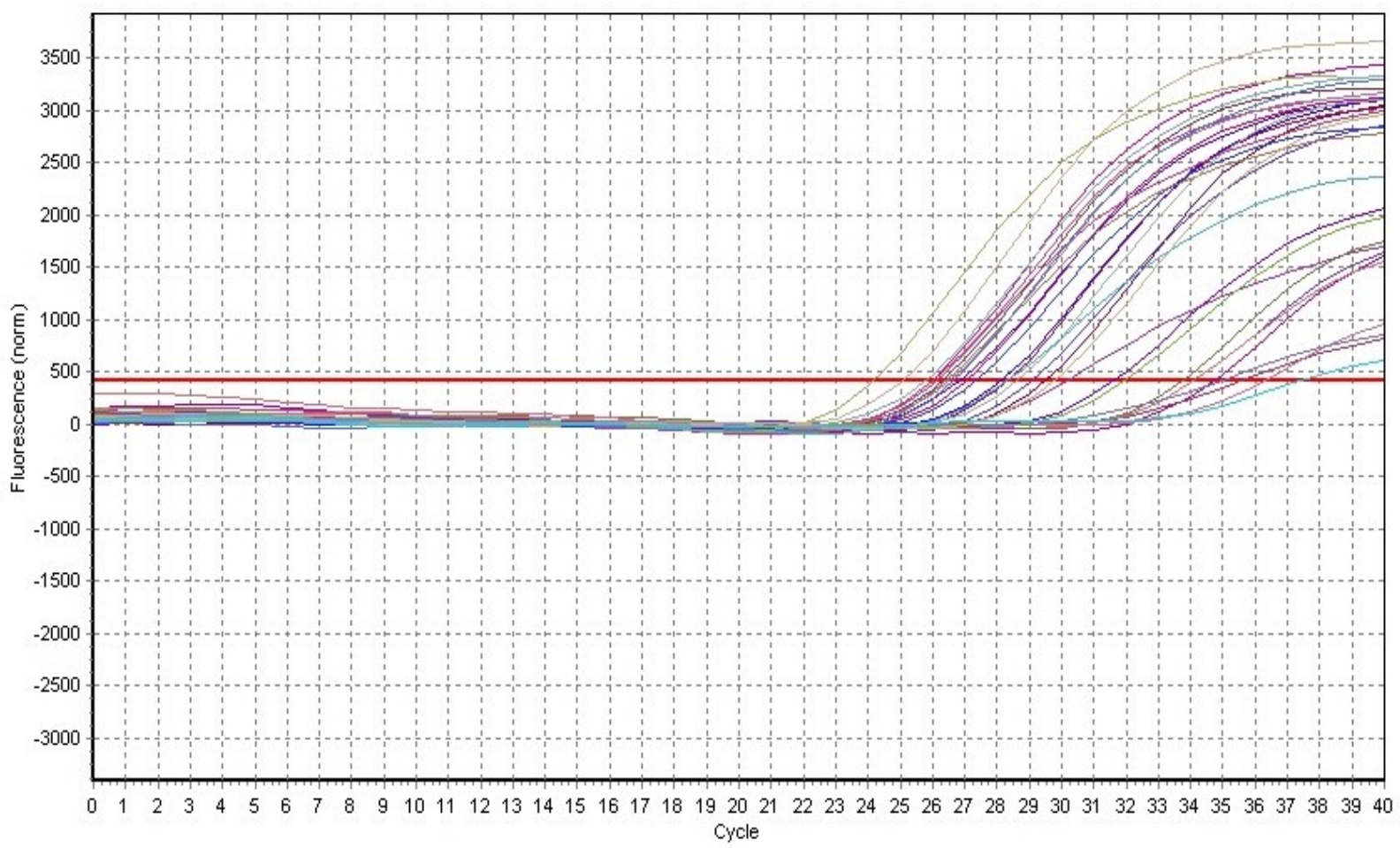

Fig. 6. TaqMan-MGB real-time RT-PCR analysis of clinical samples (left to right: faecal samples 1-30 and negative control)

\section{Discussion}

Since the 1980s, few endemic PED cases have been reported; however, since autumn of 2010, a new mutated PED virus (PEDV) strain has been spreading across China. This strain induces a classical clinical syndrome, is highly contagious, and is responsible for high mortality rate of newborn piglets reaching as high as $90 \%$ (9). Several mutations were identified in this strain at the beginning of the outbreaks; consequently, it is difficult to detect PEDV in clinical samples using conventional primers for PCR. This has hindered the prevention and control of PED (18). The aim of this study was to develop a method for more specific and accurate detection of classical and epidemic PEDV strains in China. Based on an alignment of seven different PEDV strains and using the PEDV ZJCZ4 sequence as a template, we designed a pair of primers and a probe within the conserved sequence of the $\mathrm{N}$ gene. We then used these reagents to develop a TaqMan-MGB real-time RT-PCR method with high sensitivity, specificity, and repeatability for the detection of PEDV.

To date, techniques for PEDV detection are mainly based on conventional PCR (24), nested PCR (25), LAMP-PCR (20), and real-time RT-PCR. Conventional PCR and nested PCR methods are of low-sensitivity and are therefore often inefficient for the detection of PEDV in clinical samples. Although LAMP-PCR has a higher sensitivity and can be performed using any PCR apparatus, it is less specific than real-time RT-PCR (7).

The most distinct difference between conventional PCR and real-time PCR is that with the fluorescent chemicals, signals of accumulation of amplified gene product can be detected by real-time PCR system. By comparing with standard curve, it is possible to quantify amplified molecules of the targeted gene (14).

There are two types of real-time PCR, based on chemical dyes or fluorescently labelled oligo- 
nucleotides probe. SYBR Green is a commonly used fluorescent dye for real-time PCR, which can specifically bind to double-stranded DNA. Binding of SYBR Green and the intensity of fluorescence is proportional to the amount of amplified doublestranded DNA during the PCR amplification (21). However, a drawback is that SYBR Green can also bind to primer-dimer or non-specific amplified DNA, resulting in false positive signals (8).

Another commonly used real-time PCR approach is TaqMan-MGB. With fluorescent group on 5' and a quenching group on 3', a specific oligo-nucleotide probe within the amplified product is introduced into real-time PCR reaction. When the structure of a probe does not breakdown, the quenching group can absorb fluorescent signals from 5', so the apparatus cannot detect the signal from the oligo-nucleotide probe. During PCR amplification, these two chemical groups on bonded probe will be cleaved by 5'to 3' exonuclease, resulting in detectable fluorescent signal. Once a PCR product is amplified, one fluorescent signal will be detected by PCR apparatus. With this property, the increase in the fluorescent signal is the same as the amount of amplified DNA molecules. By comparing with standard curve, it is possible to calculate the number of copies of the amplified PCR product (1). This method enables precise quantification of virus particles from clinical samples, by calculating the number of copies of the target gene. With low autofluorescence and background, this method is more specific than other real-time PCR probes (1). In addition, low false positive rate in fluorescent probe method as compared to SYBR Green method is observed. Therefore, in the present study, we developed a real-time RT-PCR assay using TaqMan-MGB as the probe, which proved to be a useful approach for PEDV detection.

Because the $\mathrm{N}$ gene of PEDV is conserved among different strains, it has been often chosen as a target for identification of the strain (18). Our probe of $27 \mathrm{~kb}$ was able to detect PEDV titres as low as $0.1 \mathrm{TCID}_{50}$. In comparison with conventional PCR and using the same pair of primers, we showed that the developed method has higher sensitivity.

In conclusion, the TaqMan-MGB real-time RTPCR assay developed in this study can be used to identify both classical and epidemic strains of PEDV in China, and is a useful technique available for studies of epidemiology and disease pathogenesis as well as for vaccine development.

Conflict of Interests Statement: The authors declare that there is no conflict of interests regarding the publication of this article.

Financial Disclosure Statement: This work was supported by the Natural Science Foundation of China (31472211), Natural Science Foundation of Shanghai
(14ZR1421400), and State Key Laboratory of Veterinary Biotechnology (SKLVBF201406).

Animal Rights Statement: None required.

Acknowledgements: Yan-Jun Zhou and Zhi-Biao Yang were corresponding authors, and we thank them for designing, directing, and composing this study.

\section{References}

1. Aryam M., Shergill I.S., Williamson M., Gommersall L., Arya N., Patel H.: Basic principles of real-time quantitative PCR. Expert Rev Mol Diagn 2005, 5, 209-219.

2. Carvajal I.L., Rafael D., Pedro R., Pedro G.: Seroprevalence of porcine epidemic diarrhea virus infection among different types of breeding swine farms in Spain. Prev Vet Med 1995, 23, 33-40.

3. Chen J., Wang C., Shi H., Qiu H., Liu S., Chen X., Zhang Z., Feng L.: Molecular epidemiology of porcine epidemic diarrhea virus in China. Arch Virol 2010, 155, 1471-1476.

4. Chen Q., Li G., Stasko J., Thomas J.T., Stensland W.R., Pillatzki A.E., Gauger P.C., Schwartz K.J., Madson D., Yoon K.J.: Isolation and characterization of porcine epidemic diarrhea viruses associated with the 2013 disease outbreak in US swine. J Clin Microbiol 2013, 52, 234-243.

5. Chen X., Yang J., Yu F., Ge J., Lin T., Song T.: Molecular characterization and phylogenetic analysis of porcine epidemic diarrhea virus (PEDV) samples from field cases in Fujian, China. Virus Genes 2012, 45, 499-507.

6. Fan J.H., Zuo Y.Z., Li J.H., Pei L.H.: Heterogeneity in membrane protein genes of porcine epidemic diarrhea viruses isolated in China. Virus Genes 2012, 45, 113-117.

7. Gardner S.N., Kuczmarski T.A., Vitalis E.A., Slezak T.R.: Limitations of Taq-Man PCR for detecting divergent viral pathogens illustrated by hepatitis $\mathrm{A}, \mathrm{B}, \mathrm{C}$, and $\mathrm{E}$ viruses and human immunodeficiency virus. J Clin Microbiol 2003, 41, 2417-2427.

8. Gu J.P., Yue X.W., Li C.Y., Xing R., Yuan C.L., Cui L., Hua X.G., Yang Z.B. Development and primary application of the SYBR Green I RT-qPCR assay for the detection of the transmissible gastroenteritis virus (TGEV) $\mathrm{S}$ gene. $4^{\text {th }}$ International Conference on Biomedical Engineering and Informatics (BMEI'11), 2011, Shanghai, pp. 1412-1415.

9. Huang Y.W., Dickerman A.W., Pineyro P., Li L., Fang L., Kiehne R., Opriessnig T., Meng X.J.: Origin, evolution, and genotyping of emergent porcine epidemic diarrhea virus strains in the United States. mBio 2013, 4, e00737-13.

10. Kim S.H., Kim I.J., Pyo H.M., Tark D.S., Song J.Y., Hyn B.H.: Multiplex real-time RT-PCR for the simultaneous detection and quantification of transmissible gastroenteritis virus and porcine epidemic diarrhea virus. J Virol Methods 2007, 146, 172-177.

11. Li W., Li H., Liu Y., Pan Y., Deng F., Song Y., Tang X., He Q. New variants of porcine epidemic diarrhea virus, China, 2011. Emerg Infect Dis 2012, 18, 1350-1353.

12. Li Z., Chen F., Yuan Y., Zeng X., Wei Z., Zhu L., Sun B., Xie Q., Cao Y., Xue C., Ma J., Bee Y.: Sequence and phylogenetic analysis of nucleocapsid genes of porcine epidemic diarrhea virus (PEDV) strains in China. Arch Virol 2013, 158, 1267-1273.

13. Li Z.L., Zhu L., Ma J.Y., Zhou Q.F., Song Y.H., Sun B.L., Chen R.A., Xie Q.M., Bee Y.Z.: Molecular characterization and phylogenetic analysis of porcine epidemic diarrhea virus (PEDV) field strains in south China. Virus genes 2012, 45, 181-185.

14. Oldham J.: Pig Farming (Oct suppl), 1972, 72-73.

15. Ouyang S.Y., Yang D., Ouyang H.S., Ma H.W.: Real-time fluorescent quantitative PCR technique and its application. Chem Life Sci 2004, 26, 74-76. 
16. Pan X.T., Li W., Zhou Q.F., Wang D.D., Bi Y.Z., Chen F., Song Y.H.: Isolation and characterization of a variant porcine epidemic diarrhea virus in China. Virol J 2012, 9, 195-203.

17. Park S., Kim S., Song D., Park B.: Novel porcine epidemic diarrhea virus variant with large genomic deletion, South Korea. Emerg Infect Dis 2014, 20, 2089-2092.

18. Park S.J., Song D.S., Park B.K.: Molecular epidemiology and phylogenetic analysis of porcine epidemic diarrhea virus (PEDV) field isolates in Korea. Arch Virol 2013, 158, 1533-1541.

19. Reed L.J., Muench H.: A simple method of estimating fifty per cent endpoints. Am J Epidemiol 1938, 27, 493-497.

20. Ren X., Li P.: Development of reverse transcription loopmediated isothermal amplification for rapid detection of porcine epidemic diarrhea virus. Virus Genes 2011, 42, 229-235.

21. Salin C., Thaweesak C., Mai L Q., Sudarat D., Arunee C., Alongkorn A., Olfert L., Thaweesak S., Apiradee T.B., Yong P.R. H5N1 Oseltamivir-resistance detection by real-time PCR using two high sensitivity labeled TaqMan probes. J Virol Methods 2007, 139, 44-49.
22. Song D., Park B.: Porcine epidemic diarrhoea virus: a comprehensive review of molecular epidemiology, diagnosis, and vaccines. Virus genes 2012, 44, 167-175.

23. Sun R.Q, Cai R.J., Chen Y.Q., Liang P.S., Chen D.K., Song C.X.: Outbreak of porcine epidemic diarrhea in suckling piglets, China. Emerg Infect Dis 2012, 18, 161-163.

24. Zhang K., He Q.G.: Development and application of multiple RT-PCR assay for PEDV, TGEV and PoRV. Chin. J Anim Vet Sci 2010, 41, 1001-1005.

25. Zhang S.F., Jia Y., Wang M.X., Ni Y.X., He K.W., Chen F.Y.: Establishment of nested RT-PCR diagnostic method for porcine epidemic diarrhea virus. Virol Sinica 2004, 19, 174-176.

26. Zhao P.D., Bai J., Tang T.S., Li Y.F., Tan C. Shi X.: Development of a multiplex TaqMan probe-based real-time PCR for discrimination of variant and classical porcine epidemic diarrhea virus. J Virol Methods 2014, 206, 150-155.

27. Zhou Y.J., Wu Y.L., Zhu J.P., Tong W., Yu H., Jiang Y.F., Tong G.Z:: Complete genome sequence of a virulent porcine epidemic diarrhea virus strain. J Virol 2012, 86, 13862. 